\title{
Silane-coupling effect of a silane-containing self-adhesive composite cement
}

Kumiko Yoshihara ${ }^{1,2}$, Noriyuki Nagaoka ${ }^{3}$, Yukinori Maruo ${ }^{4}$, Goro Nishigawa ${ }^{4}$, Yasuhiro Yoshida ${ }^{5}$, Bart Van Meerbeek ${ }^{6}$

1. National Institute of Advanced Industrial Science and Technology (AIST), Health Research Institute, 2217-14 Hayashi-cho, Takamatsu, Kagawa 761-0395, Japan

2. Okayama University Hospital, Center for Innovative Clinical Medicine, 2-5-1 Shikata-cho, Kita-ku, Okayama 700-8558, Japan. k-yoshi@md.okayama-u.ac.jp

3. Okayama University Dental School, Advanced Research Center for Oral and Craniofacial Sciences, 2-5-1 Shikata-cho, Kita-ku, Okayama 700-8558, Japan. nagaoka@okayama-u.ac.jp

4. Okayama University Hospital, Department of Occlusion and Removable Prosthodontics, 2-5-1 Shikata-cho, Kita-ku, Okayama 700-8558, Japan. ykmar@md.okayama-u.ac.jp (Dr. Maruo), goro@md.okayama-u.ac.jp (Dr. Nishigawa)

5. Department of Biomaterials and Bioengineering, Faculty of Dental Medicine, Hokkaido University, Kita 13, Nishi 7, Kita-ku, Sapporo, Hokkaido, 060-8586, Japan. yasuhiro@den.hokudai.ac.jp

6. KU Leuven (University of Leuven), Department of Oral Health Sciences, BIOMAT \& UZ Leuven (University Hospitals Leuven), Dentistry, Kapucijnenvoer 7 blok a bus 7001, B-3000 Leuven, Belgium. bart.vanmeerbeek@kuleuven.be

Corresponding author: Kumiko YOSHIHARA, k-yoshi@md.okayama-u.ac.jp Keywords: Silane; ceramic; cement; bond strength; NMR; dentin; electron microscopy 


\section{ABSTRACT}

Objective: Hydrofluoric-acid etching followed by silanization is a routine clinical protocol for durable bonding to glass ceramics. Simplifying ceramic-bonding procedures, new technological developments involve the inclusion of a silane coupling agent in a self-adhesive composite cement. To investigate the effectiveness of the incorporated silane coupling agent, shear bond strength (SB) to ceramic and dentin, contact angle of water (CA), transmission electron microscopy (TEM), X-ray diffraction (XRD) and ${ }^{29} \mathrm{Si}$ nuclear magnetic resonance (NMR) assessments were correlatively conducted.

Materials and Methods: SB to glass ceramic was measured without ('immediate') and with ('aged') $50 \mathrm{~K}$ thermocycles upon application of (1) the silane-containing self-adhesive composite cement Panavia SA Cement Universal ('SAU'), being light-cured: 'SAU_light', (2) 'SAU_chem': chemically cured SAU, (3) 'SAP_light': light-cured Panavia SA Cement Plus ('SAP'), and (4) 'SAP_CP': SAP light-cured after separate silanization using Clearfil Ceramic Primer Plus ('CP'). CA was also measured on glass ceramic. The cement pastes before and upon mixing were characterized using ${ }^{29} \mathrm{Si}$ NMR. SB of SAU or SAP onto dentin was measured. Finally, the cement-dentin interface was characterized by TEM and XRD.

Results: The immediate and aged SB to glass ceramic of SAU did not significantly differ from those of SAP_CP, while they were significantly higher than those of SAP. CA of SAU did not significantly differ from that of SAP_CP, but it was significantly higher than CA of SAP. ${ }^{29} \mathrm{Si}$ NMR revealed siloxane bonds after mixture. SB of SAU and SAP to dentin did not show any significant difference. SEM, TEM and XRD confirmed tight and chemical interaction, respectively.

Significance: Incorporating silane in a 10-MDP-based self-adhesive composite cement combined efficient silane-coupling ability at the ceramic surface with effective bonding ability at dentin.

\section{Highlights}

The clinical procedure to adhesively lute ceramic restorations is complex, by which a simplified application procedure is desirable.

Silane-coupling monomers degrade in water; hydrolysis is avoided when the silane-coupling monomer is added to the cement's hydrophobic paste. Once mixed, the silane-coupling monomer hydrolyses to chemically interact with the ceramic surface.

The novel silane-containing self-adhesive cement combined efficient silane-coupling ability onto glass ceramics with adequate self-adhesiveness onto dentin. 


\section{Introduction}

Dental glass ceramics, such as leucite and lithium-disilicate glass ceramics, are nowadays often used to adhesively restore teeth because of the patients' increasing demand for esthetics and the improved computer-aided-design/computer-aided-manufacturing (CAD/CAM) systems. Strongly upcoming alternative restoratives are resin-based composite CAD/CAM blocks, like they are today in Japan promoted, as restorations made from these blocks are reimbursed by the Japanese health insurance systems to reduce metal consumption [1].

The mechanisms involved mainly in bonding to dental ceramics and composites are micro-mechanical interlocking and chemical binding [2-4]. Micro-mechanical interlocking can be obtained by either etching glass-rich ceramics, commonly using hydrofluoric-acid (HF), or by sandblasting, which is preferentially recommended to provide micro-mechanical interlocking at the surface of composite CAD/CAM blocks. Upon HF etching or sandblasting, additional chemical binding to both ceramic and composite CAD/CAM restorations is achieved through silanization using dedicated ceramic primers. They contain silane coupling agents, of which $\gamma$-methacryloxypropyl trimethoxysilane ( $\nu$-MPTS) is most commonly used $[5,6]$. Silane coupling occurs when the alkoxy groups of the bi-functional silane molecule hydrolyse to silanols, upon which monomer adsorption to the ceramic/composite substrate occurs through condensation [7]. It is difficult to maintain the silanol status for a long time, limiting the shelf life of silane primers. Most commercial one-bottle and thus silane-coupling primers are supplied in a water-free solvent, upon which the silane is activated when clinically applied. To avoid having to use the rather caustic HF, even not used in certain countries, and to simplify ceramic-bonding procedures, combined etching and silanization has more recently been made possible with the introduction of Monobond Etch \& Prime (Ivoclar Vivadent, Schaan, Liechtenstein) $[2,3,8]$. Previous research showed that silane-containing so-called 'universal' adhesives did not have enough silane-coupling effect $[7,9]$. The silane-coupling monomer $\psi$-MPTS was found to have undergone condensation in the adhesive bottle because of the high acidic nature of the adhesive solution in the presence of water [7]. Therefore, separate silanization using a ceramic primer remained recommended when using such a silane-containing adhesive.

However, the clinical procedure to adhesively lute ceramic restorations, involving a multi-step surface treatment of both the tooth prep (or core build-up) and the ceramic restoration, remains complex. Therefore, there is a definite demand to simplify this application procedure. Recently, a new silane-containing self-adhesive cement was developed and introduced on the market. The silane-coupling efficiency of this silane-containing self-adhesive cement has not yet (independently) been determined.

In this study, the silane effect of the new silane-containing self-adhesive cement Panavia SA Cement Universal (Kuraray Noritake, Tokyo, Japan; 'SAU') and its bonding ability were investigated. The silane effect was tested using a conventional shear bond-strength approach in light- and self-cure mode onto glass ceramic, as compared with that upon luting with a silane-free self-adhesive cement and upon separate silanization with a ceramic primer followed by luting with the silane-free self-adhesive cement. The contact angle of water onto the same glass ceramic exposed to luting agents, with the silane-free self-adhesive cement applied with/without the separate silane primer, was measured to 
determine surface hydrophobicity/hydrophilicity. Mechanistic research involved characterization of the silane-coupling monomer status in the new cement using nuclear magnetic resonance (NMR). Finally, the bonding efficacy onto dentin was determined using shear bond-strength testing as compared to that of the silane-free self-adhesive cement with/without separate prior silanization, while the resultant cement-dentin interfaces were ultra-morphologically characterized using scanning/ transmission electron microscopy (S/TEM). The null hypotheses tested in this study were that (1) the new silane-containing cement does not have a silane-coupling effect onto glass ceramic, (2) the new silane-containing self-adhesive cement bonded less effectively to dentin than the silane-free self-adhesive cement, and both irrespective of light-curing or chemical curing.

\section{Materials and Methods}

\subsection{Shear-bond strength onto leucite glass-ceramic plates}

Leucite-based glass-ceramic IPS Empress CAD blocks (Ivoclar Vivadent; Shade A2) were cut into disks $10 \times 10 \mathrm{~mm}$ wide and $1.0 \mathrm{~mm}$ in thickness. The surface was polished using a $15 \mu \mathrm{m}$ diamond lapping film (Struers, Ballerup, Denmark) to a smooth surface, reducing the potential for mechanical micro-retention. Four different cement protocols were applied: (1) 'SAU_light': SAU cured by light, (2) 'SAU_chem': SAU chemically cured, (3) 'SAP_light': Panavia SA Cement Plus (Kuraray Noritake), a silane-free self-adhesive cement, cured by light, and (4) 'SAP_CP': Clearfil Ceramic Primer Plus (Kuraray Noritake; 'CP') applied and air-dried prior to the application of SAP, which is light-cured (Table 1). Zirconia cylinder blocks (Tosoh, Tokyo, Japan) with a 3.6- $\mathrm{mm}$ diameter were sandblasted using a Shofu High Blaster (Shofu, Kyoto, Japan) with $50 \mu \mathrm{m}$ alumina particles (Shofu), followed by silanization using Clearfil Ceramic primer Plus (Kuraray Noritake); the blocks were luted using one of the four experimental luting protocols onto the glass-ceramic disks using finger pressure (corresponding to a pressure of about $2.2 \mathrm{MPa}$ ) [10]. The cement, except for the SAU_chem experimental group when the cement was allowed to self-cure for $30 \mathrm{~min}$, was light-cured for $40 \mathrm{~s}$ from two opposing directions (totaling a 80-s curing time) using F3 mode (high-intensity blue LED mode) of a G-Light Prima II Plus (GC, Tokyo, Japan) light-emitting diode (LED) light-curing unit with a light irradiance of $2000 \mathrm{~mW} / \mathrm{cm}^{2}$. The specimens were next stored in $37^{\circ} \mathrm{C}$ water. For each experimental group, 20 specimens were prepared. Ten specimens were kept for $24 \mathrm{~h}$ prior to being subjected to a shear bond-strength test. Another 10 specimens were thermocycled ('TC': $60 \mathrm{~s}$ of immersion, alternatively, in a $5^{\circ} \mathrm{C}$ and $55^{\circ} \mathrm{C}$ water bath) for 50,000 cycles before executing the shear bond-strength test using a Shear Bond Tester (Bisco, Schaumburg, IL, USA). Fractured specimens were analyzed using a light microscope (SMZ-10, Nikon, Tokyo, Japan) to assess the fracture pattern. For statistical comparisons of the data, two-way analysis of variance (ANOVA) and Tukey post hoc tests were applied with $p<0.05$ considered statistically significant.

\subsection{Contact-angle measurement of water onto cement-treated glass ceramic}

Glass-ceramic plates were prepared in the same way as described previously for the shear bond-strength test. The ceramic plates were cleaned in an acetone ultrasonic bath, upon which they 
were treated as follows: (1) SAU applied and after $20 \mathrm{~s}$ washed using ethanol, (2) same procedure using SAP, (3) CP applied and air-dried, followed by the application of SAP and after 20 s washed using ethanol. Untreated glass ceramic served as control. The contact angle of distilled water dropped on the treated ceramic plates was measured using contact-angle measurement equipment (SImage AUTO 100, Excimer, Yokohama, Japan). Specimen images were analyzed using a computer program (SESF, Excimer) using an angular dimension tool to measure the contact angle ( $\theta$ ). Right and left angles were measured to obtain a mean $\theta$ value. All measurements were done in triplicate, after which the data were analyzed by one-way ANOVA and a Tukey multiple comparison test ( $\alpha=0.05)$.

\subsection{NMR analysis of silane reaction in SAU}

For NMR, experimental filler- and initiator-free SAU cement Pastes A and B were prepared and provided by Kuraray Noritake. Before measurement, paste $B$ was dissolved in the same volume of $\mathrm{d}$-methanol. Upon mixture of the experimental pastes $A$ and $B$, the mixed paste was kept for $3 \mathrm{~h}$ in atmosphere, upon which they were dissolved in the same volume of $d$-methanol. Each sample was poured into NMR test glass tubes with a 5-mm diameter and an 8-inch length (Wilmad, Buena, NJ, USA). An $400-\mathrm{MHz}$ NMR spectrometer (Bruker, Tokyo, Japan) was employed to acquire ${ }^{29} \mathrm{Si}$ NMR spectra at $100.58 \mathrm{MHz}$ in $\mathrm{CD}_{3} \mathrm{CD}_{2} \mathrm{OD}$.

\subsection{Shear-bond strength onto dentin}

Thirty extracted non-carious human molars (approved by the Commission for Medical Ethics of Okayama University under the file number \#1606-020) were used. The teeth were embedded in epoxy resin (EpoFix, Ballerup, Denmark). Dentin was exposed and the surface was polished using 600-grit SiC paper (WTCC-S, Nihon Kenshi, Fukuyama, Japan). This was followed by one of the following surface treatments: (1) SAU_light, (2) SAU_chem, (3) SAP_light and (4) SAP_chem. Zirconia cylinders, prepared in the same manner as for the bond-strength measurements onto glass-ceramic plates, were luted onto dentin using one of the three experimental luting protocols onto dentin and light-cured for $40 \mathrm{~s}$ from two opposing directions (totaling a 80-s curing time) using F3 mode (high-intensity blue LED mode) of G-Light Prima II Plus (Kuraray Noritake) except for the SAU/SAP_chem experimental groups, which were allowed to self-cure during $30 \mathrm{~min}$. Ten specimens were prepared for each experimental group. All specimens were subjected to a shear bond-strength testing protocol after $24-\mathrm{h}$ storage in water at $37^{\circ} \mathrm{C}$. Fractured specimens were analyzed using using Feg-SEM (JSM-6701F, Jeol, Tokyo, Japan) to assess the fracture pattern. For statistical comparisons of data, one-way ANOVA followed by Tukey's post hoc tests $(\alpha<0.05)$ was used with $p<0.05$ considered statistically significant.

\subsection{Thin-film XRD of dentin exposed to composite cements}

Dentin flat plates $(10 \times 8 \times 1 \mathrm{~mm})$ were cut from human molar teeth (approved by the Commission for Medical Ethics of Okayama University). The dentin surface was polished using 600-grit SiC-paper (WTCC-S). SAU or SAP was applied on the dentin surface. After $20 \mathrm{~s}$, the samples were washing using ethanol in order to remove the cement. The surface structures of SAU- and SAP-treated dentin 
(SAU/SAP_D) specimens were examined by thin-film XRD using an X-ray diffractometer (RINT2500, Rigaku, Tokyo, Japan) under 40-kV acceleration and 200-mA current, with the angle of the incident $X$-ray beam fixed at $1.0^{\circ}$ and a scanning time of $0.02^{\circ} / \mathrm{s}$ for a $2 \theta$ scan. Interaction of an experimental 10-methacryloyloxydecyl dihydrogen phosphate (10-MDP) solution, consisting of 15wt\% 10-MDP, $45 \mathrm{wt} \%$ ethanol and $40 \mathrm{wt} \%$ water, served as reference.

\subsection{Feg-SEM and TEM of cement-dentin interfaces}

Four extracted non-carious human third molars (approved by the Commission for Medical Ethics of Okayama University under the file number \#1606-020) were used. After removal of the occlusal crown third using a diamond saw (IsoMet 1000, Buehler, Lake Bluff, IL, USA), the exposed dentin was wet-sanded using 600-grit SiC paper (WTCC-S, Nihon Kenshi) to produce a standard smear layer. The composite cements SAU or SAP were applied onto dentin (SAU_light, SAP_light). The top of the cement was covered with matrix Tape (3M Oral Care, St. Paul, MN, USA). Each pair of specimens was light-cured for $20 \mathrm{~s}$ using the G-Light Prima Plus (GC) light-curing unit. The cement-bonded dentin specimens were then processed for Feg-SEM and TEM using TEM-specimen processing described in detail in previous work [11]. In brief, this specimen processing involved fixation using $2.5 \mathrm{wt} \%$ glutaraldehyde (Nacalai Tesque, Kyoto, Japan), staining with $2 \mathrm{wt} \%$ osmium (TAAB Laboratories Equipment, Aldermaston, UK), and gradual dehydration in ascending ethanol concentrations prior to embedding in epoxy resin (TAAB Laboratories Equipment). For cross-sectional Feg-SEM observations, specimen cross-sections were prepared using a cross-section polisher (SM-09020CP Cross Section Polisher, JEOL). Subsequently, a thin layer of osmium was deposited on their surfaces (Neo Osmium coater, Meiwa, Osaka, Japan), upon which the specimens were examined with Feg-SEM (JSM-6701F, $\mathrm{JEOL}$ ) operated at $5 \mathrm{kV}$ and employing an annular semiconductor detector. For TEM, ultrathin sections were cut (Leica EM UC7, Leica, Vienna, Austria) prior to being observed with high-resolution TEM (HR-TEM, $200 \mathrm{kV}$ ) utilizing a JEM-2100 microscope (JEOL).

\section{Results}

\subsection{Shear bond strength onto leucite glass-ceramic plates (Fig. 1)}

When the silane-containing self-adhesive composite cement SAU was light- (SAU_light) or chemically cured (SAU_chem), the resultant bond strength onto leucite glass-ceramic plates was not statistically significantly different from that recorded for the silane-free cement SAP when applied following separate silanization (SAP_CP) (Fig. 1a). Without prior silanization, a significantly lower bond strength was recorded for SAP_light. After long-term thermocycling, SAP_light presented with the significantly lowest bond strength, while no statistically significant difference in bond strength was found between SAU_light, SAU_chem and SAP_CP. Moreover, no significant decrease in bond strength was recorded upon aging among the latter three experimental groups. In contrast, when the silane-free self-adhesive composite cement SAP was applied without prior silanization, nearly no bond strength remained upon long-term TC. Bonded cement-ceramic specimens failed mostly cohesively within the ceramic for SAU_light, SAU_chem and SAP_CP with/without thermocycling, while SAU_light 
specimens failed at the actual cement-ceramic interface (Fig. 1b). 


\subsection{Contact-angle measurement of water onto cement-treated glass ceramic (Fig. 2)}

The contact angle of water on untreated glass ceramic (control) was significantly the lowest, while the highest contact angles without statistically significant difference among them were recorded for the silane-containing composite cement SAU and the silane-free cement SAP when applied following separate silanization (SAP-CP). Without prior silanization, SAP presented with a significantly lower contact angle than that of SAU and SAP_CP, and a significantly higher contact angle than that of the control.

\subsection{NMR analysis of silane reaction in SAU (Fig. 3)}

When the silane-coupling monomer was added to the experimental cement paste ${ }^{B},{ }^{29} \mathrm{Si}$ NMR disclosed one sharp peak at $-42.9 \mathrm{ppm}$, which was assigned to the methoxy groups of the silane-coupling monomer. Mixture of the experimental pastes $A$ and $B$ revealed after $3 \mathrm{~h}$ an additional peak at $-51.6 \mathrm{ppm}$, which was assigned to $\mathrm{Si}-\mathrm{O}-\mathrm{Si}$ (siloxane).

\subsection{Shear bond strength onto dentin (Fig. 4)}

No statistically significant difference in bond strength onto dentin was recorded among all experimental groups SAU-light, SAU_chem, SAP_light and SAP_chem. Most bonded cement-dentin specimens failed adhesively at the actual interface for all experimental groups, as evidenced by bur scratches visible at all dentin surfaces (Fig. 4b).

\subsection{Thin-film XRD of dentin exposed to composite cements (Fig. 5)}

Interaction of the silane-containing composite cement SAU and the silane-free cement SAP resulted in three characteristic peaks, which upon comparison with the 10-MDP reference were assigned to 10-MDP_Ca salt formation, indicative of nano-layering.

\subsection{Feg-SEM and TEM of cement-dentin interfaces (Figs. 6 and 7)}

Feg-SEM and TEM interfacial characterization of the SAU_light- and SAP_light-dentin interfaces showed a porosity-free tight interface.

Feg-SEM identified filler in different sizes and shapes for both composite cements (Fig. 6). No smear-layer remnants were observed at the interfaces of SAU_light and SAP_light with dentin. High-magnification Feg-SEM of the SAU_light-dentin interface disclosed a $500-\mathrm{nm}$ to $1-\mu \mathrm{m}$ thick hybrid layer versus a 500-nm thick hybrid layer for the SAP_light-dentin interface.

TEM confirmed the SEM observations (Fig. 7). Both cements produced around 500-nm thick hydroxyapatite (HAp)-rich hybrid layers, with a slightly thicker hybrid layer observable at the SAU_light-dentin interface. High-magnification TEM revealed an approximately 3.6-nm nano-layered structure, more abundantly for SAU_light than for SAP_light.

\section{Discussion}

This study investigated the silane-coupling effect and bonding ability onto both glass-ceramic and 
dentin of a newly developed silane-containing self-adhesive composite cement. The rationale behind this product development was to incorporate silane into the composite cement to simplify the multi-step adhesive luting protocol commonly used for HF-etched glass-ceramic and alumina-sandblasted composite restorations. A previous attempt to simplify this adhesive luting procedure, involving the incorporation of silane within universal adhesives, appeared less successful $[7,9]$. The latter failure should basically be ascribed to the instability of the silane-coupling monomer in the acidic adhesive solution of universal adhesives. Hence, the current study aimed to investigate if the new silane-containing self-adhesive cement is as effective as that of a self-adhesive composite cement that is applied following separate silanization using a ceramic primer.

Silane-coupling monomers can chemically bond through their silanol groups to form covalent -Si-O-Si- bonds with glass [12]. In this study, the silane-coupling effect of the new silane-containing cement SAU was evaluated onto leucite-based glass ceramic, because previous research revealed that silane effectively coupled onto leucite-based glass-ceramic IPS Empress CAD blocks [13, 14]. The latter was confirmed in this study with SAP_CP that, involving separate silane treatment prior to the application of the self-adhesive cement, resulted in the highest bond strength to the glass-ceramic blocks at $24 \mathrm{~h}$ and upon 50k TC. The bond-promoting effect of such separate silanization for glass-ceramics appeared also from other research [5, 15]. For silane to bond to glass, the silane-coupling monomer needs to be activated by hydrolysis with water to turn their methoxy groups into silanols $[6,7]$. Besides the silane-coupling monomer, the ceramic primer CP used in this study contains the acidic functional monomer 10-MDP in ethanol; CP does not contain water. The silane-coupling monomer in this ceramic primer reacts with water in the atmosphere. Unlike a ceramic primer, the composite cement SAU consists of two high-viscosity pastes that need to be mixed prior to their application. As pastes cannot readily 'catch' water, it is very doubtful that the silane-coupling monomers in the cement paste could hydrolyze during the clinical application time. In case of SAU_light, the cement was cured immediately upon luting and seating the zirconia cylinder onto the glass-ceramic plate. In contrast with the light-curing mode, which increases the degree of conversion immediately upon light irradiation $[16,17]$, the polymerization reaction of the chemical curing mode is however relatively slow $[16,17]$. Because of this contrasting difference in polymerization rate, SAU was applied following both the light- and chemical curing protocols in order to investigate the effect of reaction time of the silane-coupling monomer on glass ceramic. Nevertheless, both SAU_chem and SAU_light revealed a statistically similar bond strength as was obtained with SAP_CP when luting was preceded by separate silanization. The first null hypothesis that the new silane-containing cement does not have a silane-coupling effect onto glass ceramic, this irrespective of light-curing or chemical curing, was rejected. This means that the in-build silane in SAU was sufficiently effective when applied following a common light-curing clinical application procedure. It is probable that water adsorbed onto glass hydrolyzed the silane-coupling monomers in the cement [18].

As silanization is especially thought relevant for bond durability, the absence of significant decrease in bond strength after thermocycling additionally confirms that the silane coupling of SAU was effective. Indeed, both SAU_light as SAU_chem revealed durable bonding onto glass ceramics that 
was not different from that recorded for SAP_CP involving prior separate silanization. Fracture-mode analysis upon shear bond-strength testing clearly confirmed the silane-coupling effect of SAU. SAU_light and SAU_chem revealed cohesive fractures within ceramic, as recorded also for SAP_CP, indicating that effective chemical binding with the ceramic surface was obtained. On the other hand, SAP_light revealed cement-ceramic interfacial fractures, indicative of inferior interfacial bonding.

In addition to effective silane coupling, the degree of polymerization conversion also affects bond strength. Some dual-cure cements were reported to insufficiently chemically cure, even after $24 \mathrm{~h}$ [16]. However, other dual-cure composite cements, such as RelyX U200 (3M Oral Care), Clearfil SA Luting (Kuraray Noritake) and G-Cem Link Ace (GC) revealed a similar compressive strength upon chemical curing as light-curing [19]. As in this study the shear bond strength was also tested after 24 $\mathrm{h}$ and no significant difference in bond strength between SAU_chem and SAU_light was recorded, it can be concluded that SAU has a favorable chemical curing ability.

The silane effect in SAU was also evaluated using water contact-angle measurement. Contact-angle measurement is often used to confirm the silane-coupling effect [12]. When silane is adsorbed on a glass surface, the methacrylate group at the other end of the bi-functional silane monomer is positioned at the outer glass surface [20], hereby increasing the surface's hydrophobicity. In this study, the cement was washed off using ethanol in order to evaluate the surface wettability of the cement-treated glass ceramics. Washing with a polar solvent, such as ethanol, removes the physically deposited but not the chemically adsorbed monomers [21]. The chemically adsorbed silane-coupling monomers will serve as receptors for co-polymerization with other methacrylates, such as bisphenol A-glycidyl methacrylate (Bis-GMA), diurethane dimethacrylate (UDMA), etc., commonly being present in adhesives and cements [22]. Separate silanization as part of SAP_CP resulted in high surface hydrophobicity that was not significantly different from that measured upon the application of the silane-containing self-adhesive composite cement SAU. These data indicate that SAU and SAP_CP produced a monolayer of silane-coupling monomers chemically adsorbed onto glass ceramic. While the glass-ceramic surface exposed to the silane-free composite cement SAP became also more hydrophobic as compared to untreated glass ceramic, it did not reach the hydrophobicity of glass ceramic exposed to SAU and SAP_CP. Overall, the contact-angle results agreed with the bond-strength data. The manufacturer's data sheet showed that SAU contains a long-carbon-chain silane (trimethoxysilyl long-chain alkyl methacrylate), referred to as 'LCSi' (technical information from Kuraray Noritake). CP however contains the more commonly used silane monomer $\gamma$-MPTS. Although the monomer length of LCSi is longer than that of $\psi$-MPTS, no significant change in contact angle was found.

Based on the shear bond-strength data onto glass ceramic and the contact-angle, the first null hypothesis that the new silane-containing cement does not have a silane-coupling effect onto glass ceramic, failed to be accepted.

To examine the chemical status of the silane-coupling monomer in the ceramic paste and upon mixing the two pastes, ${ }^{29} \mathrm{Si} N \mathrm{NM}$ was employed. ${ }^{29} \mathrm{Si} \mathrm{NMR}$ provides qualitative and quantitative information about the hydrolysis and self-condensation reaction [23]. Alternatively, ${ }^{1} \mathrm{H}$ and ${ }^{13} \mathrm{C} \mathrm{NMR}$ have also been used to investigate both silane-coupling monomer reactions [24, 25]. Most studies 
analyzed simple formulation compositions, often containing a silane-coupling monomer, acidic monomer and solvent $[24,25]$. Later, ${ }^{1} \mathrm{H}$ and ${ }^{13} \mathrm{C}$ NMR techniques were used to detect the hydrolysis reaction of silane in adhesives $[7,9]$. In the latter studies, solely 4 or $10 \mathrm{wt} \%$ silane was added to commercial adhesives, by which the peaks assigned to the silane-coupling monomer were quite small $[7,9]$. However in this study, self-adhesive cements, such as SAU, contain methacrylate monomers in high concentration in addition to silica filler without solvent. Pilot studies using ${ }^{1} \mathrm{H} N M R$ and ${ }^{13} \mathrm{C} N M R$ revealed that peaks assigned to silane-coupling monomers could hardly be detected. Therefore, ${ }^{29} \mathrm{Si}$ NMR was instead used in this study. However, using ${ }^{29} \mathrm{Si} \mathrm{NMR,} \mathrm{peaks} \mathrm{representing} \mathrm{silica} \mathrm{filler} \mathrm{were}$ found to overlap with the peaks disclosing silane-coupling monomers. Hence, experimental filler-free SAU Pastes A and B were analyzed by NMR in this study. Also the polymerization initiator was omitted so that upon mixing Pastes $A$ and $B$ polymerization did not occur during measurement. Finally, silane-coupling monomer was added to the experimental composite-cement formulations to clearly detect silane.

${ }^{29} \mathrm{Si}$ NMR of Paste B revealed one single strong peak at $-41.0 \mathrm{ppm}$, which was assigned to the methoxy groups of the silane-coupling monomer [23]. After Pastes A and B were mixed, another significant peak at $-50 \mathrm{ppm}$ in addition to the $-41.0 \mathrm{ppm}$ peak was detected. The ${ }^{29} \mathrm{Si} \mathrm{NMR}$ analysis in this study involved a $7 \mathrm{~h}$ integration time before clear peaks could be detected. During NMR measurement of the cement mixture, the silane-coupling monomer started to hydrolyze and undergo self-condensation, so a new peak at -50 ppm representing self-condensation was detected. Although silanol should have been formed upon mixing Pastes $A$ and $B$, it was however not possible to detect it directly. Nevertheless, as two peaks representing both methoxy groups and -Si-O-Si- due to self-condensation were detected, silanol should have existed. According to manufacturer's technical information (Kuraray Noritake), Paste A contains hydrophilic monomers, such as 2 hydroxyethyl methacrylate (HEMA), and the functional monomer 10-MDP, while Paste $B$ has a relatively hydrophobic monomer content. The silane-coupling monomer was added to the relatively hydrophobic and $\mathrm{pH}$-neutral Paste $\mathrm{B}$ so that the silane-coupling monomer would not self-hydrolyze in the paste.

While the previously discussed shear bond-strength and contact-angle data already confirmed the silane-coupling effectiveness of the silane incorporated in the self-adhesive composite cement, ${ }^{29} \mathrm{Si}$ NMR of Paste B did not reveal the -50 ppm peak, hereby confirming that the silane-coupling monomer did not self-condensate and must have been sufficiently active upon mixture. Note that the acidic functional monomer 10-MDP may have acted as an accelerator for hydrolysis, as was mentioned before [9]. The schematic diagram showed the activation and interaction of silane monomer coupling monomers with glass in Fig 8 (1)-(4). When Paste A and Paste B kept separately (Fig.8 (1) and (2)), silane coupling monomers (LCSi) do not show hydrolysis. When Paste A and Paste B are mixed, silane coupling monomers (LCSi) hydrolysis to silanol in Fig. 8 (3). Then the mixed cement paste apply on glass, silane coupling monomers (LCSi) absorbed on glass and bond to glass in Fig. 8 (4).

A self-adhesive composite cement should not only bond to the glass-ceramic restoration, but also to the tooth. Therefore, also the bonding ability of SAU to dentin was measured in this study. A 
statistically similar bond strength to dentin was measured for the silane-containing SAU and the silane-free SAP composite cement. Both SAU and SAP are self-adhesive cements and contain 10-MDP as functional monomer. A previous study reported that the 10-MDP-containing cements G-CEM Automix (GC) and Clearfil SA cement (Kuraray Noritake) resulted in a higher bond strength to dentin than the self-adhesive cement SmartCem2 (Dentsply Sirona, Konstanz, Germany) that does not contain 10-MDP [26]. Furthermore, the 10-MDP-containing self-adhesive cement Clearfil SA cement (Kuraray Noritake) presented with a favorable immediate and aged micro-tensile bond strength, the latter measured upon two-year water storage [27].

Bonding effectiveness can be measured using several bond-strength tests, as most commonly macro- and micro-shear bond strength, and macro- and micro-tensile bond strength are measured [28]. In simulation of a clinically executed luting procedure, zirconia cylinders were in this study pressed onto ceramic/dentin surfaces, upon which the shear-bond strength was measured. Rather than a more technique-sensitive micro-tensile bond-strength test, a shear-bond strength test was selected to not induce collateral stress at the adhesive interface, in particular when the macro-specimens would have been cut in multiple micro-specimens, which must have affected the bond-strength outcome. In addition, pressing the restoration during luting was thought required to achieve adequate wetting of the self-adhesive composite cement onto the substrate surfaces, hereby avoiding interfacial voids [29]. Both macro- and micro-bond strength tests provide relevant bonding effectiveness data that should be interpreted in the correct context, while also their limitations should be considered [30, 31]. Having measured the bonding/luting performance of two self-adhesive composite cements onto dentin, further tests should be conducted including also a considered gold-standard adhesive-assisted composite cement.

In this study, SAU was applied using both a light-curing (SAU_light) and chemical curing (SAU_chem) protocol. No significant difference in bond strength to dentin was recorded for SAU_light and SAU_chem, indicating that SAU possessed a favorable chemical curing ability that did not affect its self-adhesiveness.

Overall, the second hypothesis tested that the new silane-containing self-adhesive cement bonded less effectively to dentin than the silane-free self-adhesive cement, also failed to be accepted.

The functional monomer 10-MDP is known to have a strong chemical interaction ability with HAp [32]. In addition, when 10-MDP-based primer and adhesives are applied on dentin, 10-MDP was documented to self-assemble into so-called nano-layering of 10-MDP_Ca salts [33-37]. To examine the formation of 10-MDP_Ca salts, thin-film XRD was employed. Thin-film XRD was before used to analyze the interaction of functional monomers with the surface of HAp, enamel and/or dentin [36-38]. However, the methodology employed before to detect 10-MDP_Ca salt formation at the substrate surface needed adaptation in this study. To be able to examine the surface of dentin that was exposed to the cements, the cements were washed off using ethanol. XRD revealed three characteristics peaks representative for 10-MDP_Ca salt formation for both the silane-containing and silane-free self-adhesive composite cements SAU and SAP, respectively. To form 10-MDP_Ca salts, Ca should be released from dentinal HAp [33]. The fact that 10-MDP_Ca salt formation was detected proved that the functional monomer 10-MDP present in the cement pastes could react with HAp in 
dentin. The schematic diagram showed the interaction of 10-MDP on tooth in Fig 8 (5) and (6). 10-MDP can reacts with Ca of tooth HAp (5), and formed 10-MDP Ca salts (6).

Feg-SEM and TEM cement-dentin interfacial characterization confirmed that both cements formed a tight interface formation with dentin, while no residual smear fragments were detected [26] They produced an about $500 \mathrm{~nm}$ thick hybrid layer with that produced by SAU being slightly thicker than the hybrid layer produced by SAP. This could indicate that SAU must have interacted deeper/more intensively with dentin than SAP. Nevertheless, it did not result in significantly different bonding effectiveness. Most bonded cement-dentin specimens failed adhesively near the interface for all experimental groups, as evidenced by scratches visible at all dentin surfaces (Fig. 4b). High magnification nevertheless revealed cement fragments that remained attached to the dentin surface. For SAP_chem, some interfacial porosities can be observed at certain areas. Such porosities have been described before, in particular when the composite cement was solely allowed to chemically cure (no light-curing), by which the relatively slow curing process promoted water uptake from the underlying dentin through osmosis [39]. High-magnification TEM interfacial characterization ultra-morphologically confirmed nano-layering of 10-MDP_Ca salts for both SAU and SAP, hereby in agreement with the XRD data. Nano-layering appeared slightly more intense for SAU than for SAP, this in agreement with the slight difference in hybrid-layer thickness reported above. To form 10-MDP_Ca salts, 10-MDP needs to etch HAp, forcing dentin to release Ca that then should be 'caught' by 10-MDP to form nano-layers [33]. The functional monomer 10-MDP is somewhat unique to have a relatively high etching efficacy among several functional monomers tested and used in different dental adhesives and cements [40]. Importantly, the water resistance of 10-MDP_Ca salts is thought to contribute to the long-term bond stability [41].

A long-term aged bond-strength test should now be conducted to evaluate bond durability.

\section{Conclusion}

In this study, we tested the silane-coupling effect onto glass ceramic as well as the bonding ability to dentin of the newly developed silane-containing self-adhesive cement SAU. Investigating first the shear bond strength with/without thermocycling revealed that SAU was equally effective to bond to glass ceramic as the silane-free self-adhesive cement that was applied following prior separate silanization. In contrast to previous unfavorable findings regarding the incorporation of silane into universal adhesives, adding silane to the hydrophobic paste of SAU appeared successful, enabling to simplify the adhesive luting procedure that no longer needs separate silanization. Silane incorporated in the self-adhesive composite cement worked effectively because the silane-coupling monomer did not undergo hydrolysis condensation when it was added to the hydrophobic paste and not to the acidic hydrophilic paste.

\section{Acknowledgment}

Funding/conflict of interest: This study was supported by JSPS KAKENHI [grant number: JP 
18K17068].The authors declare that they have no conflict of interest in this research. We thank Kuraray Noritake Dental for providing the experimental cement.

\section{References}

[1] Yoshihara K, Nagaoka N, Maruo Y, Nishigawa G, Irie M, Yoshida Y, Van Meerbeek B. Sandblasting may damage the surface of composite CAD-CAM blocks. Dent Mater 2017;33:e124-e135. doi.org/10.1016/j.dental.2016.12.003

[2] Dimitriadi M, Zinelis S, Zafiropoulou M, Silikas N, Eliades G, Self-Etch Silane Primer: Reactivity and Bonding with a Lithium Disilicate Ceramic, Materials (Basel) 2020; 13641. doi.org/10.3390/ma13030641

[3] Prado M, Prochnow C, Marchionatti AME, Baldissara P, Valandro LF, Wandscher VF. Ceramic Surface Treatment with a Single-component Primer: Resin Adhesion to Glass Ceramics. J Adhes Dent. 2018;20(2):99-105. doi: 10.3290/j.jad.a40303.

[4] Peumans M, Valjakova EB, De Munck J, Mishevska CB, Van Meerbeek B, Bonding Effectiveness of Luting Composites to Different CAD/CAM Materials, J Adhes Dent 18(4) (2016) 289-302. doi: 10.3290/j.jad.a36155.

[5] Tian T, Tsoi JK, Matinlinna JP, Burrow MF. Aspects of bonding between resin luting cements and glass ceramic materials.Dent Mater 2014;30:e147-62. doi: 10.1016/j.dental.2014.01.017.

[6] Matinlinna JP, Lung CYK, Tsoi JKH. Silane adhesion mechanism in dental applications and surface treatments: A review. Dent Mater 2018;34:13-28. https://doi.org/10.1016/j.dental.2017.09.002

[7] Yoshihara K, Nagaoka N, Sonoda A, Maruo Y, Makita Y, Okihara T, Irie M, Yoshida Y, Van Meerbeek

B. Effectiveness and stability of silane coupling agent incorporated in 'universal' adhesives. Dent Mater 2016;32:1218-25. https://doi.org/10.1016/j.dental.2016.07.002

[8] Scherer MM, Prochnow C, Venturini AB, Pereira GKR, Burgo TAL, Rippe MP, Valandro LF.Fatigue failure load of an adhesively-cemented lithium disilicate glass-ceramic: Conventional ceramic etching vs etch \& prime one-step primer. Dent Mater. 2018;34:1134-1143. doi: 10.1016/j.dental.2018.04.012. [9] Yao C, Yu J, Wang Y, Tang C, Huang C. Acidic pH weakens the bonding effectiveness of silane contained in universal adhesives. Dent Mater 2018;34: 809-818. https://doi.org/10.1016/j.dental.2018.02.004.

[10] Nagaoka N, Yoshihara K, Feitosa VP, Tamada Y, Irie M, Yoshida Y, Van Meerbeek B, Hayakawa S. Chemical interaction mechanism of 10-MDP with zirconia. Sci Rep. 2017; 30;7:45563. doi: 10.1038/srep45563.

[11] Van Meerbeek B, Yoshida Y, Lambrechts P, Vanherle G, Duke ES, Eick JD, Robinson SJ. A TEM study of two water-based adhesive systems bonded to dry and wet dentin. J Dent Res 1998;77:50-9. https://doi.org/10.1177/00220345980770010501

[12] Lung CY, Matinlinna JP. Aspects of silane coupling agents and surface conditioning in dentistry: an overview. Dent Mater 2012;28:467-77. https://doi.org/10.1016/j.dental.2012.02.009

[13] Lee Y, Chae M, Kim KH, Kwon TY. Effect of dental silane primer activation time on resin-ceramic bonding. J Adhes Sci Technol 2015;29:1155-67. https://doi.org/10.1080/01694243.2015.1022498 
[14] Peumans M, Valjakova EB, De Munck J, Mishevska CB, Van Meerbeek B. Bonding Effectiveness of Luting Composites to Different CAD/CAM Materials. J Adhes Dent. 2016;18:289-302. doi: 10.3290/j.jad.a36155.

[15] Li RW, Chow TW, Matinlinna JP. Ceramic dental biomaterials and CAD/CAM technology: state of the art. J Prosthodont Res. 2014;58:208-16. https://doi: 10.1016/j.jpor.2014.07.003.

[16] Aguiar TR, de Oliveira M, Arrais CA, Ambrosano GM, Rueggeberg F, Giannini M. The effect of photopolymerization on the degree of conversion, polymerization kinetic, biaxial flexure strength, and modulus of self-adhesive resin cements. J Prosthet Dent 2015;113:128-34. https://doi.org/10.1016/j.prosdent.2014.09.011

[17] Lührs AK, Pongprueksa P, De Munck J, Geurtsen W, Van Meerbeek B. Curing mode affects bond strength of adhesively luted composite CAD/CAM restorations to dentin. Dent Mater 2014;30:281-91. https://doi.org/10.1016/j.dental.2013.11.016

[18] Kahn FJ. Orientation of liquid crystals by surface coupling agents Appl Phys Lett 1973;22:386-8, https://doi.org/10.1063/1.1654684

[19] Lee Y, Kim JH, Woo JS, Yi YA, Hwang JY, Seo DG. Analysis of Self-Adhesive Resin Cement Microshear Bond Strength on Leucite-Reinforced Glass-Ceramic with/without Pure Silane Primer or Universal Adhesive Surface Treatment. Biomed Res Int 2015;2015:361893. http://dx.doi.org/10.1155/2015/361893

[20] Kurth DG, Bein T. Surface reactions on thin layers of silane coupling agents, Langmuir 1993;9:2965-73. https://doi.org/10.1021/la00035a039

[21] Aida M, Tabei N, Kimoto S, Tanimura H, Takahashi H, Yaguchi T, Nishiyama N. Ceramic bond durability and degradation mechanism of commercial gamma-methacryloxypropyl trimethoxysilane-based ceramic primers. Am J Dent 2012;25:231-4.

[22] Kato T, Kimura H, Saigo K, Yamada B, Yamauchi J, Anraku T. Effects of a silane coupling agent on the tensile adhesive strength between resin and titanium. J Appl Polym Sci 2013; 129: 2922-30. https://doi.org/10.1002/app.38917

[23] Salon MC, Abdelmouleh M, Boufi S, Belgacem MN, Gandini A. Silane adsorption onto cellulose fibers: hydrolysis and condensation reactions J Colloid Interface Sci. 2005:289: 249-61. https://doi.org/10.1016/j.jcis.2005.03.070

[24] Nishiyama N, Horie K, Asakura T, Hydrolysis and condensation mechanisms of a silane coupling agent studied by $13 \mathrm{C}$ and 29Si NMR. J Appl Polym Sci. 1987; 34:1619-30. https://doi.org/10.1002/app.1987.070340421

[25] Brochier Salon MC, Bayle PA, Abdelmouleh M, Boufi S, Belgacem MN. Kinetics of hydrolysis and self condensation reactions of silanes by NMR spectroscopy. Colloids Surf A 2008; 312:83-91. https://doi.org/ 10.1016/j.colsurfa.2007.06.028

[26] Suyama Y, de Munck J, Cardoso MV, Yamada T, Van Meerbeek B. Bond durability of self-adhesive composite cements to dentine. J Dent. 2013;41:908-17. https://doi.org/10.1016/j.jdent.2013.07.008 [27] Aguiar TR, André CB, Correr-Sobrinho L, Arrais CA, Ambrosano GM, Giannini M. Effect of storage times and mechanical load cycling on dentin bond strength of conventional and self-adhesive resin luting cements. J Prosthet Dent. 2014;111:404-10. http://doi: 10.1016/j.prosdent.2013.07.016. 
[28] De Munck J, Mine A, Poitevin A, Van Ende A, Cardoso MV, Van Landuyt KL, Peumans M, Van Meerbeek B. Meta-analytical review of parameters involved in dentin bonding. J Dent Res 2012;91:351-7. doi: 10.1177/0022034511431251.

[29] De Munck J, Vargas M, Van Landuyt K, Hikita K, Lambrechts P, Van Meerbeek B. Bonding of an auto-adhesive luting material to enamel and dentin. Dent Mater 2004;20:963-71.

[30] Armstrong S, Geraldeli S, Maia R, Raposo LH, Soares CJ, Yamagawa J, Adhesion to tooth structure: a critical review of "micro" bond strength test methods. Dent Mater 2010;26:e50-62. oi.org/10.1016/j.dental.2009.11.155.

[31] Braga RR, Meira JB, Boaro LC, Xavier TA, Adhesion to tooth structure: a critical review of "macro" test methods. Dent Mater 2010;26:e38-49. doi: 10.1016/j.dental.2009.11.150.

[32] Yoshida Y, Nagakane K, Fukuda R, Nakayama Y, Okazaki M, Shintani H, Inoue S, Tagawa Y, Suzuki K, De Munck J, Van Meerbeek B. Comparative study on adhesive performance of functional monomers.vJ Dent Res. 2004;83454-8. https://doi.org/10.1177/154405910408300604

[33] Yoshihara K, Yoshida Y, Nagaoka N, Fukegawa D, Hayakawa S, Mine A, Nakamura M, Minagi S, Osaka A, Suzuki K, Van Meerbeek B. Nano-controlled molecular interaction at adhesive interfaces for hard tissue reconstruction. Acta Biomater. 2010;6:3573-82. doi: 10.1016/j.actbio.2010.03.024.

[34] Yoshihara K, Yoshida Y, Hayakawa S, Nagaoka N, Irie M, Ogawa T, Van Landuyt KL, Osaka A, Suzuki $\mathrm{K}$, Minagi S, Van Meerbeek B. Nanolayering of phosphoric acid ester monomer on enamel and dentin. Acta Biomater. 2011;7:3187-95. doi: 10.1016/j.actbio.2011.04.026.

[35] Yoshihara K, Yoshida Y, Nagaoka N, Hayakawa S, Okihara T, De Munck J, Maruo Y, Nishigawa G, Minagi S, Osaka A, Van Meerbeek B. Adhesive interfacial interaction affected by different carbon-chain monomers. Dent Mater. 2013;29:888-97. doi: 10.1016/j.dental.2013.05.006.

[36] Yoshida Y, Yoshihara K, Nagaoka N, Hayakawa S, Torii Y, Ogawa T, Osaka A, Van Meerbeek B. Self-assembled Nano-layering at the Adhesive interface. J Dent Res. 2012;91:376-81. doi: $10.1177 / 0022034512437375$.

[37] Yoshihara K, Nagaoka N, Yoshida Y, Van Meerbeek B, Hayakawa S. Atomic level observation and structural analysis of phosphoric-acid ester interaction at dentin. Acta Biomater. 2019 Oct 1;97:544-556. doi: 10.1016/j.actbio.2019.08.029.

[38] Yoshihara K, Nagaoka N, Hayakawa S, Okihara T, Yoshida Y, Van Meerbeek B. Chemical interaction of glycero-phosphate dimethacrylate (GPDM) with hydroxyapatite and dentin. Dent Mater. 2018;34:1072-1081. doi: 10.1016/j.dental.2018.04.003.

[39]Lührs AK, De Munck J, Geurtsen W, Van Meerbeek B. Composite cements benefit from light-curing. Dent Mater. 2014;30:292-301. doi: 10.1016/j.dental.2013.11.012.

[40] Yoshihara K, Hayakawa S, Nagaoka N, Okihara T, Yoshida Y, Van Meerbeek B. Etching Efficacy of Self-Etching Functional Monomers. J Dent Res. 2018 Aug;97(9):1010-1016. doi: 10.1177/0022034518763606.

[41] Yoshihara K, Nagaoka N, Yoshida Y, Van Meerbeek B, Hayakawa S. Atomic level observation and structural analysis of phosphoric-acid ester interaction at dentin. Acta Biomater. 2019 Aug 16. pii: S1742-7061(19)30585-9. doi: 10.1016/j.actbio.2019.08.029. 
Table 1. List of materials investigated

\begin{tabular}{lrl}
\hline Composite cement & Paste & Composition \\
\hline Panavia SA Cement Plus (SAP) & A $\begin{array}{l}\text { Monomer (10-MDP, Bis-GMA, TEGDMA, HEMA, other } \\
\text { methacrylate monomer), filler (silanated barium glass } \\
\text { filler, silanated colloidal silica), initiator, pigment, others } \\
\text { Methacrylate monomer, filler (silanated barium glass filler, } \\
\text { silanated sodium fluoride), accelerator, pigment, others }\end{array}$ \\
\hline Panavia SA Universal (SAU) & A $\begin{array}{l}\text { Monomer (10-MDP, Bis-GMA, TEGDMA, HEMA, other } \\
\text { methacrylate monomer), filler (silanated barium glass } \\
\text { filler, silanated colloidal silica), initiator, pigment, others } \\
\text { Methacrylate monomer, filler (silanated barium glass filler, } \\
\text { aluminium oxide, silanated sodium fluoride), accelerator } \\
\text { pigment, silane coupling agent, others }\end{array}$ \\
\hline $\begin{array}{l}\text { Clearfil Ceramic Primer Plus } \\
\text { 10-MDP: 10-Methacryloyloxydecyl } \\
\text { diglycidylmethacrylate; HEMA: 2-hydroxyethyl methacrylate; TEGDMA: triethylene glycol } \\
\text { dimethacrylate. }\end{array}$ & Silane coupling agent, monomer (10-MDP), ethanol \\
\hline dihydrogen phosphate; Bis-GMA: bisphenol A
\end{tabular}




\section{Figure legends:}

Fig. 1. (a) Shear bond strength (SBS) of the silane-containing self-adhesive composite cement Panavia SA Universal (Kuraray Noritake; 'SAU'), when applied following a light-curing protocol (SAU_light) and following a chemically curing protocol (SAU_chem), and of the silane-free self-adhesive composite cement Panavia SA Cement Plus (Kuraray Noritake; 'SAP'), when applied without (SAP_light) and with prior separate silanization (SAP_CP) using Clearfil Ceramic Primer Plus (Kuraray Noritake; 'CP') onto leucite-based glass-ceramic IPS Empress CAD (Ivoclar Vivadent) blocks. Bars denote the mean bond strength with the whiskers defining the standard deviation. Inside the bars, the mean SBS value and the standard deviation are mentioned. Means with the same letter are not significantly different ( $p>0.05$ ). (b) Photomicrographs illustrating failed fractured surfaces representative for the different experimental groups.

Fig. 2. Mean contact angles measured by deposition of a water drop onto the leucite-based glass-ceramic IPS Empress CAD (Ivoclar Vivadent) blocks upon their exposure to the self-adhesisve composite cements SAU and SAP, the latter also upon prior separate silanization (SAP_CP). Untreated glass ceramic served as control. The cements were washed off using ethanol immediately prior to contact-angle measurement. Bars denote the mean contact angle with the whiskers defining the standard deviation. Inside the bars, the mean contact-angle value and the standard deviation in brackets are mentioned. Means with the same letter are not significantly different $(p>0.05)$.

Fig. 3. ${ }^{29} \mathrm{Si}$ NMR spectra representing the experimental SAU Paste $B$ in (a) and the mixed experimental SAU Pastes A and B in (b). Strong peak at $-42.9 \mathrm{ppm}$ ( $\mathbf{n}$ ), which was assigned to the methoxy groups of the silane-coupling monomer in (a) and (b) and an additional peak at $-51.6 \mathrm{ppm}(\boldsymbol{\nabla})$, which was assigned to Si-O-Si (siloxane) in (b).

Fig. 4. (a) Shear bond strength (SBS) of the silane-containing self-adhesive composite cement SAU, when applied following a light-curing protocol (SAU_light) and following a chemically curing protocol (SAU_chem), and of the silane-free self-adhesive composite cement SAP onto dentin. Bars denote the mean bond strength with the whiskers defining the standard deviation. Inside the bars, the mean SBS value and the standard deviation are mentioned. Means with the same letter are not significantly different ( $p>0.05$ ). (b) SEM photomicrographs illustrating failed fractured surfaces representative for the different experimental groups. High magnification nevertheless revealed cement fragments that remained attached to the dentin surface for all experimental groups. For SAP_chem, some interfacial porosities can be observed at certain areas (arrows), which most likely represent water droplets absorbed from the underlying dentin through osmosis.

Fig. 5. XRD of the interaction of the silane-containing composite cement SAU and the silane-free cement SAP with dentin resulted in three characteristic peaks, which upon comparison with the 10-MDP reference were assigned to 10-MDP_Ca salt formation, indicative of nano-layering. 
Fig. 6. Field-emission-gun scanning electron microscopy (Feg-SEM) of the SAU_light-dentin interface in (a-c) and the SAP_light-dentin interface in (d-f), revealing tight interfaces between cement and dentin. About 500-nm thick hybrid layers were produced, with the hybrid layer produced by SAU in (c) appearing slightly thicker than that produced by SAP in (f).

Fig. 7. Transmission electron microscopy (TEM) observation of the SAU_light-dentin interface in (a-c) and the SAP_light-dentin interface in ( $d-f)$, revealing tight interfaces between cement and dentin. About 500-nm thick hybrid layers were produced, with the hybrid layer produced by SAU in (c) appearing slightly thicker than that produced by SAP in (f). 3.6-nm nano-layering of 10-MDP_Ca salts can be observed at both the SAU_light-dentin interface in (c) and the SAP_light-dentin interface in (f), with nano-layering slightly being more abundant for SAU_light.

Fig. 8. Schematic diagram of the interaction of SAU with glass and tooth. When paste $A$ and paste $B$ are kept separately, silane coupling monomers (LCSi) in Paste B don't hydrolyze in (1) and (2). When paste $A$ and paste $B$ are mixed, LCSi hydrolyze to silanol in (3). When the mixture paste applies to glass, LCSi adsorbs on glass and promote dehydration condensation on glass in (4). When the mixture paste applies on tooth, 10-MDP monomers react with Ca of tooth HAp and form 10-MDP-Ca salts. 


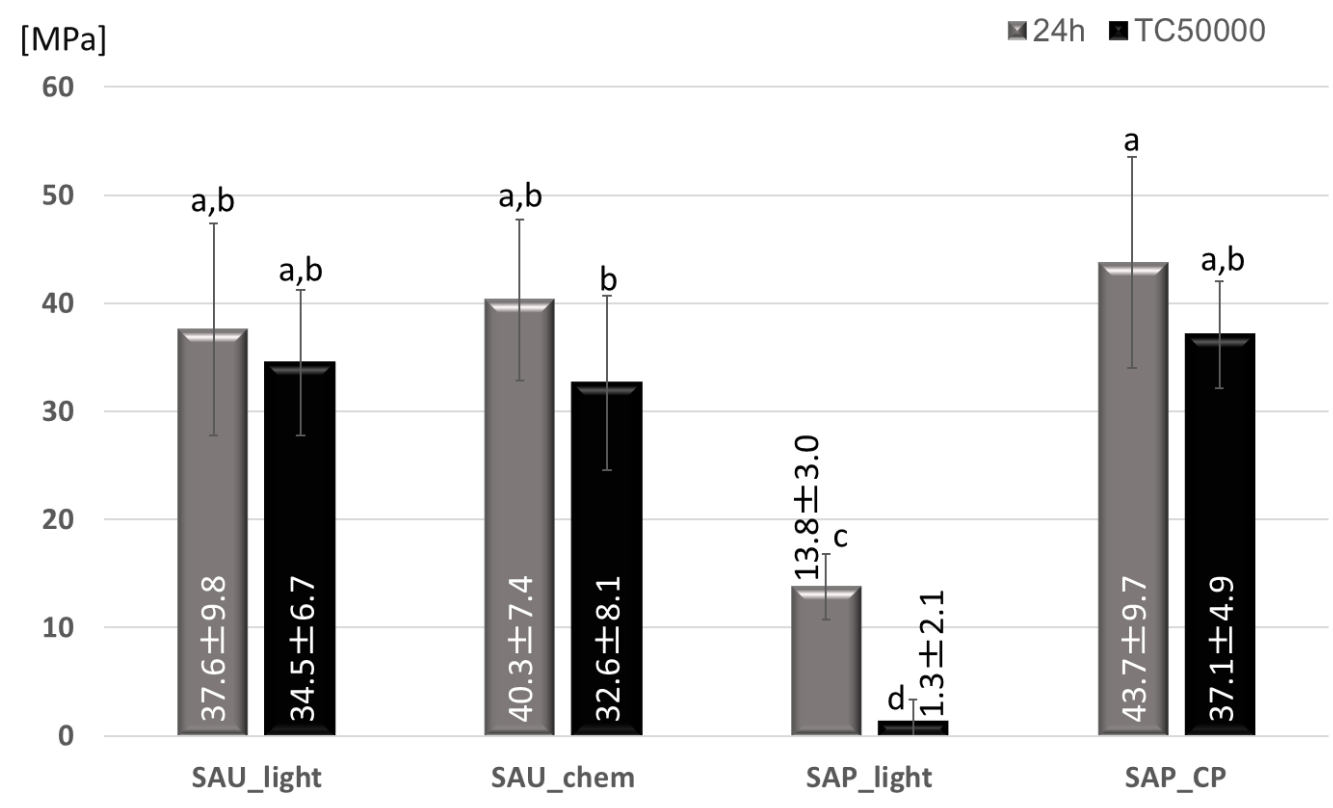

Figure 1a



Figure $1 b$ 


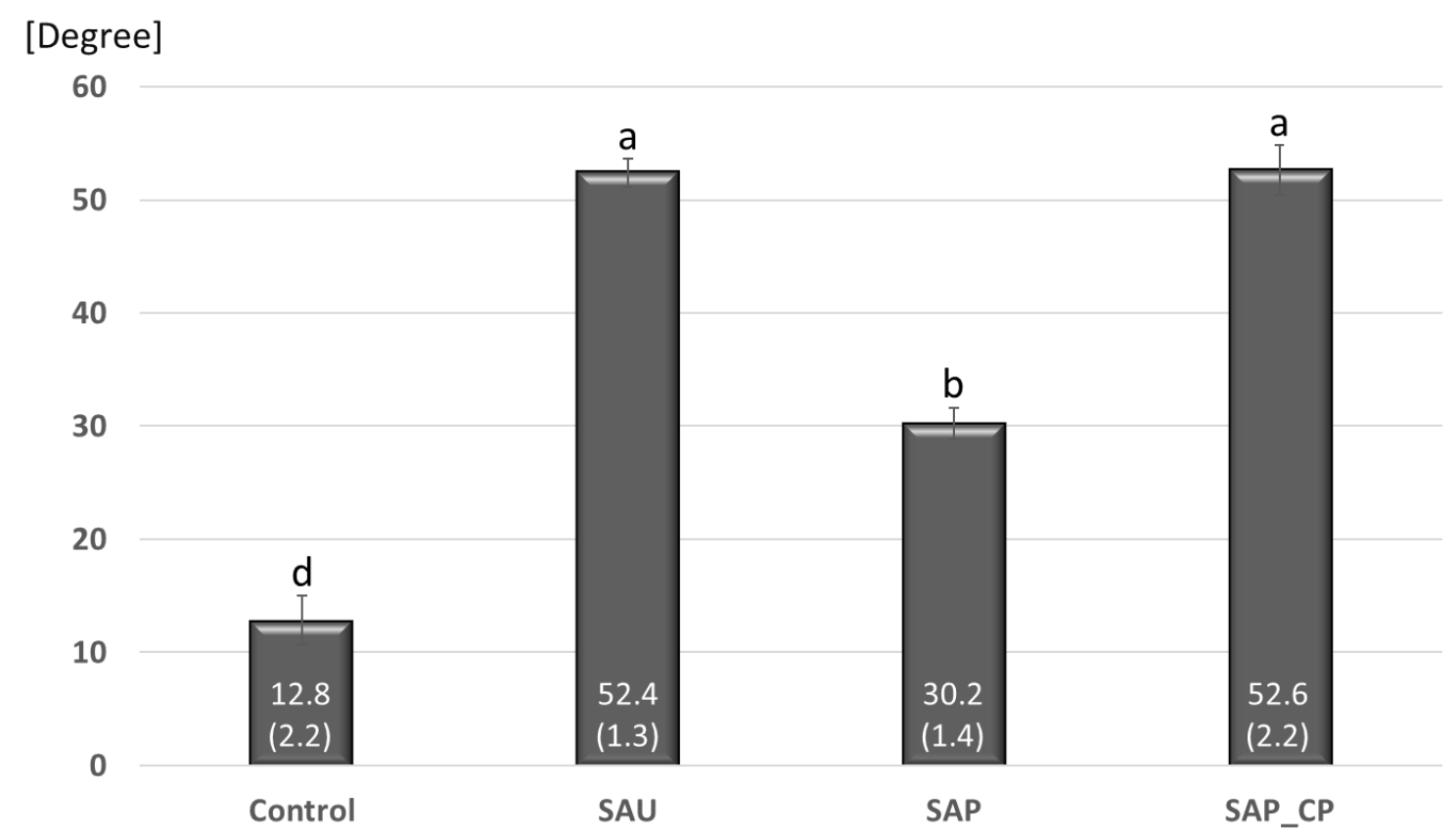

Figure 2 

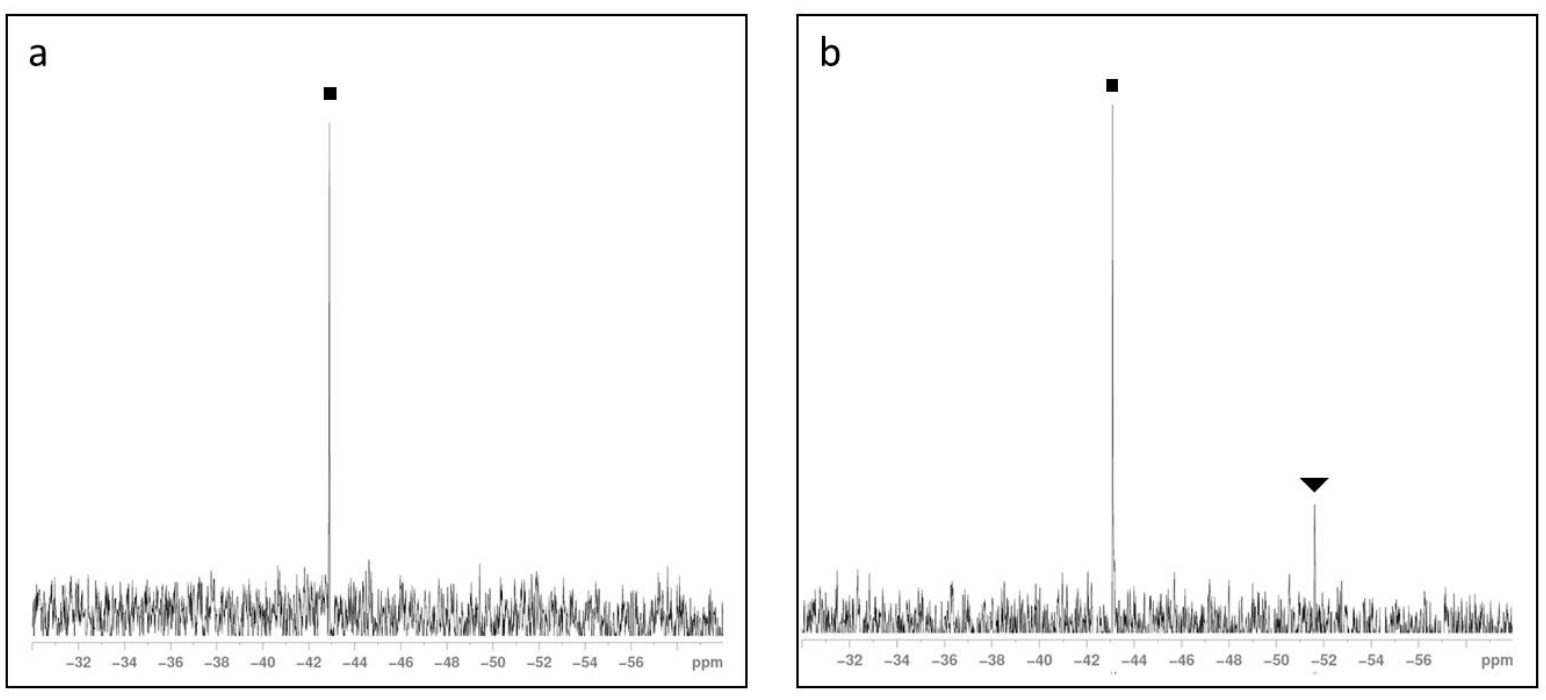

Figure 3 


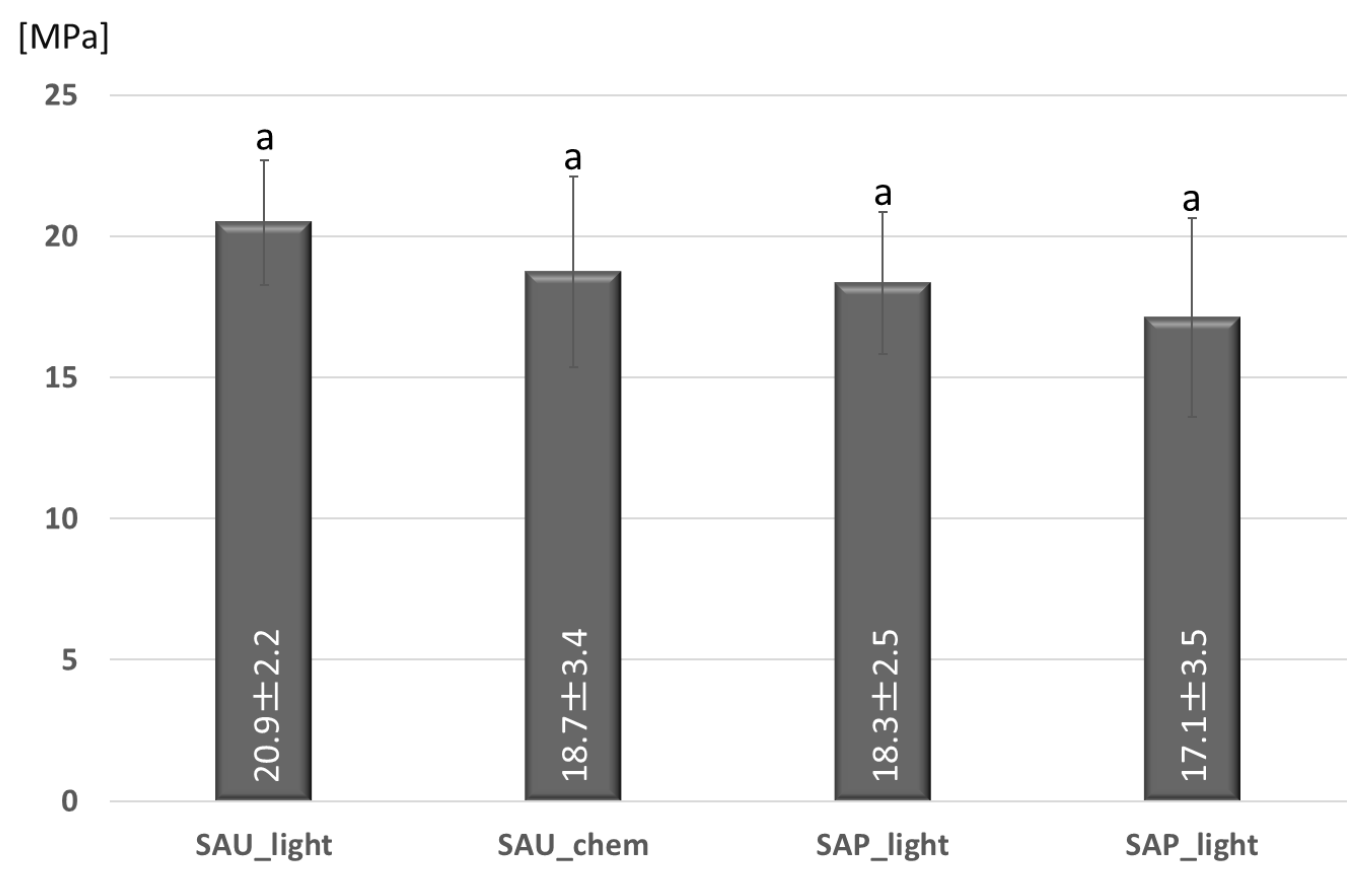

Figure 4a

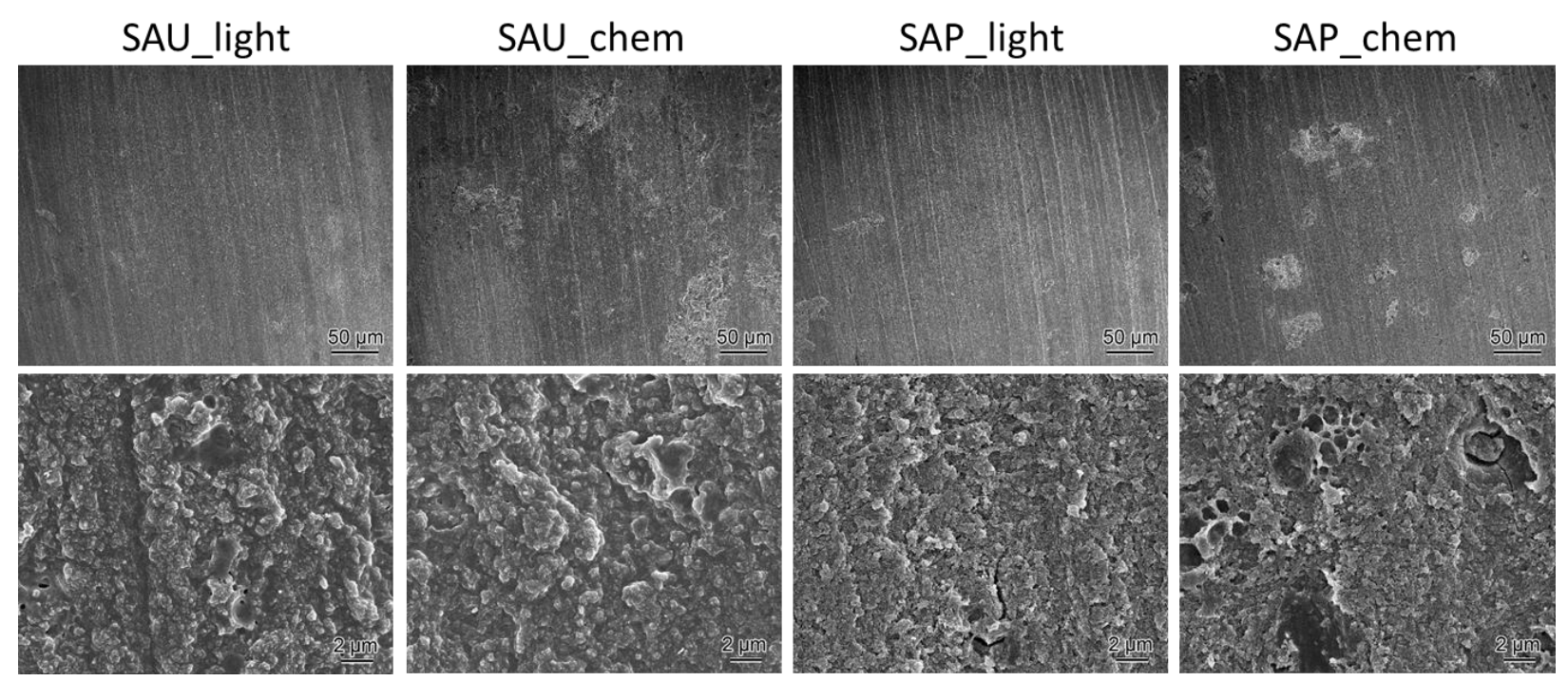

Figure4b 


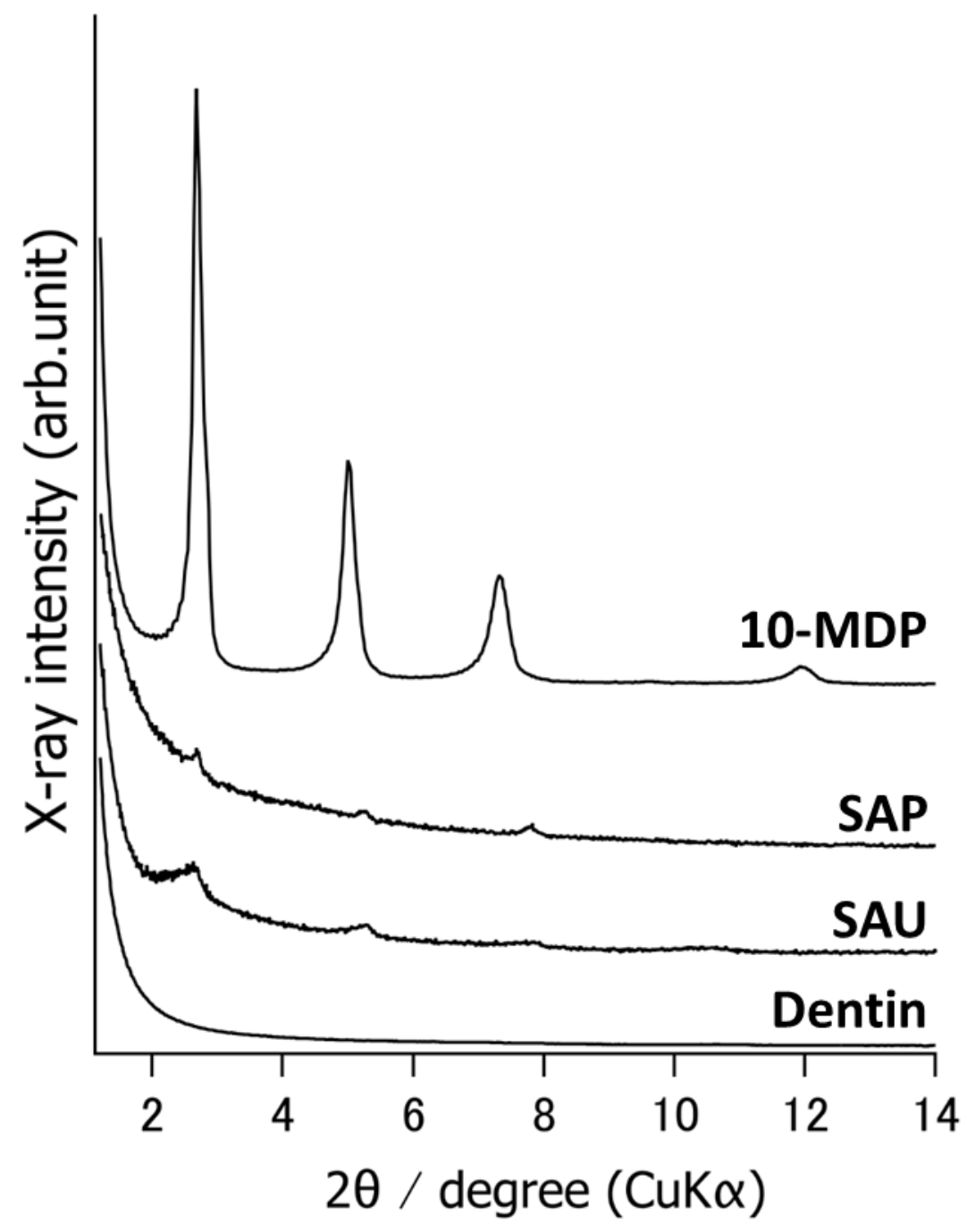

Figure5 
SAU_light

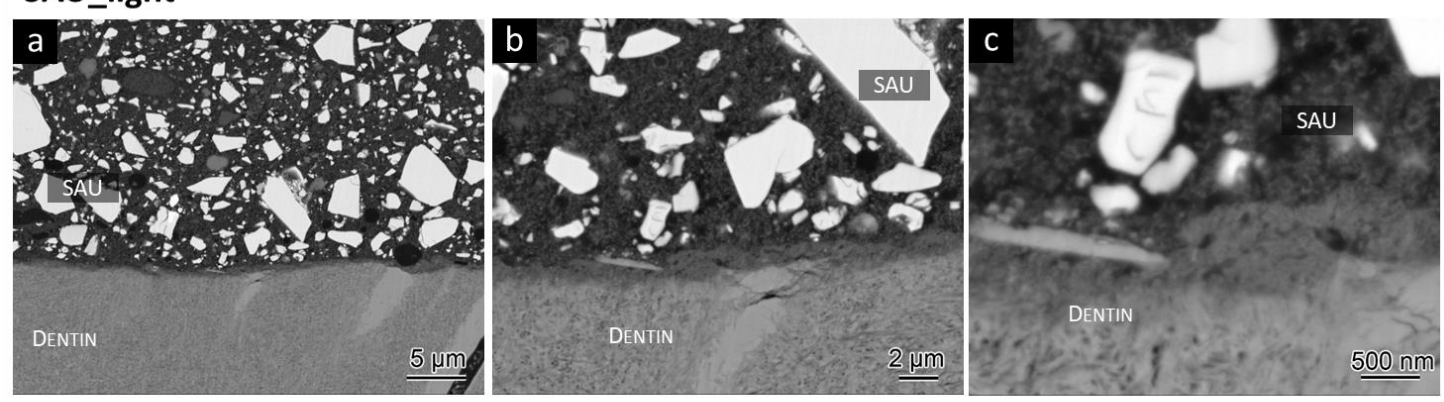

SAP_light



Figure 6 



Figure7 


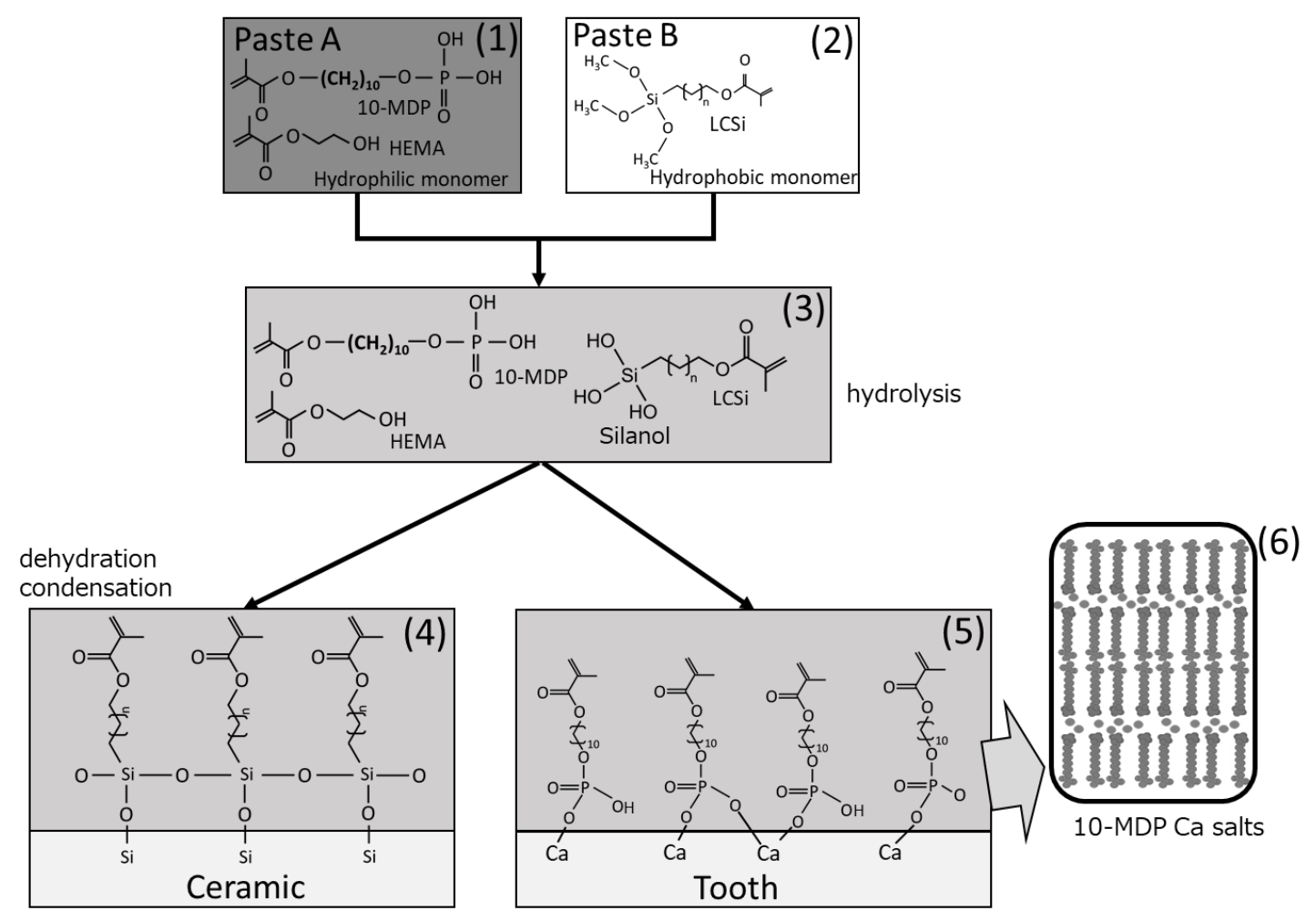

Figure 8 